\title{
Stabilization of LSD1 by deubiquitinating enzyme USP7 promotes glioblastoma cell tumorigenesis and metastasis through suppression of the $\mathbf{p 5 3}$ signaling pathway
}

\author{
LEI YI, YAN CUI, QINGFU XU and YUGANG JIANG \\ Department of Neurosurgery, The Second Xiangya Hospital of Central South University, \\ Changsha, Hunan 410011, P.R. China
}

Received April 8, 2016; Accepted August 22, 2016

DOI: $10.3892 /$ or.2016.5099

\begin{abstract}
The substrates and mechanisms of ubiquitin specific peptidase 7 (USP7) in glioma remain unclear. Lysine-specific demethylase 1 (LSD1) may undergo proteasomal degradation; however, a reciprocal mechanism that stabilizes LSD1 in glioma has not been dertermined. Here co-immunoprecipitation and GST pull-down assays revealed that LSD1 is associated with USP7 in vivo and in vitro. USP7 inhibited LSD1 ubiquitination and stabilized LSD1 in A172 and T98G cells. MTT, EdU proliferation, flow cytometry and Transwell assays indicated that LSD1 played a critical role in the proliferation and invasion of glioblastoma (GBM) cells. We defined the mechanism of USP7 in GBM, through counterbalanced LSD1 ubiquitylation. USP7 caused G0/G1 arrest, promoted tumorigenesis and invasion of A172 and T98G cells. We also uncovered the suppression of the p53 signaling pathway that mediated the activity of USP7 and LSD1. Furthermore, USP7 and LSD1 expression levels were higher in the 150 glioma patients than these levels in normal brain tissues and were correlated with glioma progression. LSD1 was increased concurrently with USP7 during glioblastoma progression and both were predictors for worsened prognosis. Collectively, our study suggested that USP7-LSD1 affects GBM cell proliferation and invasion and may be valuable as novel therapeutic targets and prognostic tools for GBM.
\end{abstract}

\section{Introduction}

Lysine-specific demethylase 1 (LSD1/KDM1) was the first discovered histone demethylase that removed the mono-methyl

Correspondence to: Professor Yugang Jiang, Department of Neurosurgery, The Second Xiangya Hospital of Central South University, 139 Renmin Middle Road, Changsha, Hunan 410011, P.R. China

E-mail: yugangjiang1964@163.com

Key words: glioblastoma, USP7, LSD1, deubiquitination, p53, tumorigenesis, metastasis and di-methyl moieties from $\mathrm{H} 3-\mathrm{K} 4$ via a flavin adenine dinucleotide (FAD)-dependent monoamine oxidoreductase and governed transcription regulation by serving as an epigenetic coregulator $(1,2)$. In previous studies, the function of LSD1 appeared to be as a tumor suppressor or oncogene depending on the tumor type. For example, overexpression of LSD1 was noted in neuroblastoma (3), non-small cell lung cancer (4), colorectal cancer (5) and prostate cancer (6) and was found to promote proliferation, migration and invasion. While in breast cancer, LSD1 was downregulated and inhibited tumor metastasis by the suppression of TGF $\beta(7,8)$. Thus, it is reasonable that the function of LSD1 is opposing due to the distinct target genes in different types of cancer. As reported, inhibition of HDACs and LSD1 are potential combination therapies in glioblastoma multiforme (GBM) (9), but the function and post-transcription of LSD1 remain unclear.

Deubiquitylating enzymes (DUBs) are a number of proteins that antagonize ubiquitylation by cleaving polyubiquitin or monoubiquitin and thus afford the stabilization or transcription regulation of the substrates. Ubiquitin specific peptidase 7 (USP7), also known as human herpesvirus-associated ubiquitin-specific protease (HAUSP) is a member of the DUB family and was first revealed to deubiquitinate and stabilize p53 $(10,11)$. Subsequently, numerous proteins have been reported as potential substrate/binding partners of USP7, such as Epstein-Barr nuclear antigen 1 (EBNA1) (12), PTEN (13), INK4a (14), the transcription factors FOXO4 (15) and REST (16). USP7 is involved in the cell cycle by the regulation of many proteins, as well as tumor suppressors or oncogenes. However, the expression pattern and substrates for USP7 in glioma patients are still unclear.

Glioma is the most common primary aggressive malignant brain tumor, among which, GBM is well known as the highest grade glioma and one of the most lethal forms of cancer in humans (17). Glioblastoma therapy, over the past decade, which combines surgery, postoperative chemotherapy and radiation therapy has failed to benefit all patients suffering from disease progression, equally. The overall median survival is still less than 15 months $(18,19)$, thus individualized therapy such as targeting several aberrant epigenetic functions should be considered as a potentially valuable approach for glioma patients. 


\section{Materials and methods}

Patients and tissue samples. The study was approved by the Research Ethics Committee of Central South University. Human brain samples were obtained from 150 patients who underwent surgical treatment at the Second Xiangya Hospital of Central South University, Changsha, China. Written informed consent was obtained from all the patients. The fresh glioma specimens were obtained between January 2008 and December 2012. The patients who had received radiotherapy or chemotherapy prior to surgery were excluded. Sections of the specimen were snap frozen and stored at $-80^{\circ} \mathrm{C}$ for mRNA isolation; other sections of the specimen were used for histological sectioning. Additionally, 10 cases of normal brain tissue samples were obtained from patients who underwent surgery for decompression treatment due to severe head injuries other than malignancies. All the glioma samples were verified by the World Health Organization (WHO) 2007 classification standard. The details of the patients are presented in Table I. In the follow-up period, overall survival was calculated from diagnosis to death; the total follow-up period was 60 months.

Cell culture and transfection. The human A172 and T98G GBM cells were obtained from the American Type Culture Collection (ATCC; Manassas, VA, USA). The aforementioned cells were cultured in Dulbecco's modified Eagle's medium (DMEM) with 10\% fetal bovine serum (FBS) (Gibco, Los Angeles, CA, USA) and incubated at $37^{\circ} \mathrm{C}$ in humidified $5 \% \mathrm{CO}_{2}$. When the cells were grown to $70 \%$ confluence, relative plasmids or siRNAs were transfected into the cells using Lipofectamine 2000 reagent (Invitrogen Life Technologies, Carlsbad, CA, USA) according to the manufacturer's instructions. The siRNAs and the negative controls were synthesized by Shanghai GenePharma (Shanghai, China). The knockdown efficiency was measured $48 \mathrm{~h}$ after transfection.

Real-time PCR. Total RNA was extracted from the glioma specimens or the control normal brain tissues or the GBM cells using TRIzol reagent (Invitrogen Life Technologies). The concentration and purity of RNA were determined, and then $\sim 2 \mu$ g RNA was used to synthesize cDNA using the cDNA Reverse Transcription kit (TransGen Biotech, Inc., Beijing, China). Real-time PCR reaction amplification was performed using the SYBR-Green PCR Master Mix on a 7500 Fast RealTime PCR system cycler (Applied Biosystems, Foster City, CA, USA). The expression of the relative genes was analyzed using the $2^{-\Delta \Delta C t}$ method. GAPDH mRNA was used as an internal control to normalize the selected genes in the same sample. The amplification protocol consisted of denaturation at $98^{\circ} \mathrm{C}$ for $5 \mathrm{~min}$, followed by 40 cycles of denaturation at $98^{\circ} \mathrm{C}$ for $10 \mathrm{sec}$ and annealing and extension at $60^{\circ} \mathrm{C}$ for $30 \mathrm{sec}$. The process was repeated for 40 cycles.

Co-immunoprecipitation assay. For co-immunoprecipitation assay (co-IP), the cells were washed with cold phosphatebuffered saline (PBS) and lysed with cold lysis buffer $(50 \mathrm{mM}$ Tris-HCl pH 7.4, 0.5\% SDS, $150 \mathrm{mM} \mathrm{NaCl,} 1 \% \mathrm{NP}-40$, and $1 \mathrm{mM}$ EDTA) at $4^{\circ} \mathrm{C}$ for $45 \mathrm{~min}$, followed by centrifugation at
$132,000 \mathrm{rpm}$ for $15 \mathrm{~min}$ at $4^{\circ} \mathrm{C}$. The supernatant was collected and incubated with appropriate primary antibodies or normal rabbit/mouse immunoglobin $\mathrm{G}$ ( $\mathrm{IgG}$ ) as a negative control, on a rotator overnight at $4^{\circ} \mathrm{C}$. After being incubated with protein A/G Sepharose CL-4B beads for $2 \mathrm{~h}$ at $4^{\circ} \mathrm{C}$, the beads were washed 5 times with lysis buffer and the immune complexes were subjected onto SDS-PAGE, followed by western blotting detection.

GST pull-down assay. The GST fusion construct was expressed in BL21 Escherichia coli cells, and the in vitro transcription and translation experiments were performed using rabbit reticulocyte lysate (TNT systems; Promega) according to the manufacturer's recommendation. In the GST pull-down assay, $10 \mu \mathrm{g}$ of GST fusion protein was incubated with $10 \mu \mathrm{l}$ of the in vitro transcribed/translated products at room temperature for $30 \mathrm{~min}$. Thirty microliters of glutathione-Sepharose beads was then added to the binding reaction and mixed at $4^{\circ} \mathrm{C}$ for $2 \mathrm{~h}$. After being washed three times with binding buffer, the immune complexes were resolved by SDS-PAGE, followed by western blot detection.

Western blotting. Total proteins were purified from the GBM cells and equal amount of protein lysate $(30 \mu \mathrm{g})$ was separated on an $8 \%$ polyacrylamide gel (Invitrogen Life Technologies). Then the gel was transferred onto a PVDF membrane at $400 \mathrm{~mA}$ for $1 \mathrm{~h}$. After being blocked with 5\% non-fat milk, the membrane was incubated with the primary antibody against USP7 (sc-30164, 1:1,000; Santa Cruz Biotechnology, Inc.) or LSD1 (ab17721, 1:1,000; Abcam) or actin (1:2,000; Beyotime Institute of Biotechnology) overnight at $4^{\circ} \mathrm{C}$. Finally, the membrane was incubated with horseradish peroxidase-conjugated goat anti-rabbit or goat anti-mouse antibody at room temperature for $1 \mathrm{~h}$. The ECL detection system (BeyoECL Plus; Beyotime Institute of Biotechnology) was used to visualize the immunoblots.

Cell cycle analysis. The cell cycle was analyzed by flow cytometry (FCM). GBM cells were synchronized by serum starvation for $24 \mathrm{~h}$ and released with 10\% FBS for an appropriate period of time, and then the cells were harvested, washed with PBS, and fixed with $70 \%$ ethanol at $4^{\circ} \mathrm{C}$ overnight. Cells were incubated with RNase (Sigma-Aldrich) in PBS for $30 \mathrm{~min}$ at $37^{\circ} \mathrm{C}$ for $30 \mathrm{~min}$. Cell cycle data were collected with FACSCalibur flow cytometer (Becton-Dickinson) and analyzed with FlowJo 7.6 software. Each experiment was performed in triplicate.

Cell proliferation analysis. The cell proliferation analysis was performed using the 3-(4,5-dimethylthiazol-2-yl)-2,5-diphenyltetrazolium bromide (MTT) assay. The cells were plated into a 12 -well culture plate $(10,000$ cells/well), and incubated in a humidified atmosphere of $5 \% \mathrm{CO}_{2}$ at $37^{\circ} \mathrm{C}$. At $24,48,72$ or $96 \mathrm{~h}, 10 \mu \mathrm{l}$ was added to each well (MTT; Sigma-Aldrich), and then incubation was carried out at $37^{\circ} \mathrm{C}$ for $4 \mathrm{~h}$. Dimethyl sulfoxide (DMSO) (Sigma-Aldrich) was used to terminate the reaction. The optical absorbance was measured using a microplate reader (Bio-Rad, Hercules, CA, USA) at $570 \mathrm{~nm}$ and quantification of the cell viability was determined according to the optical density values. 

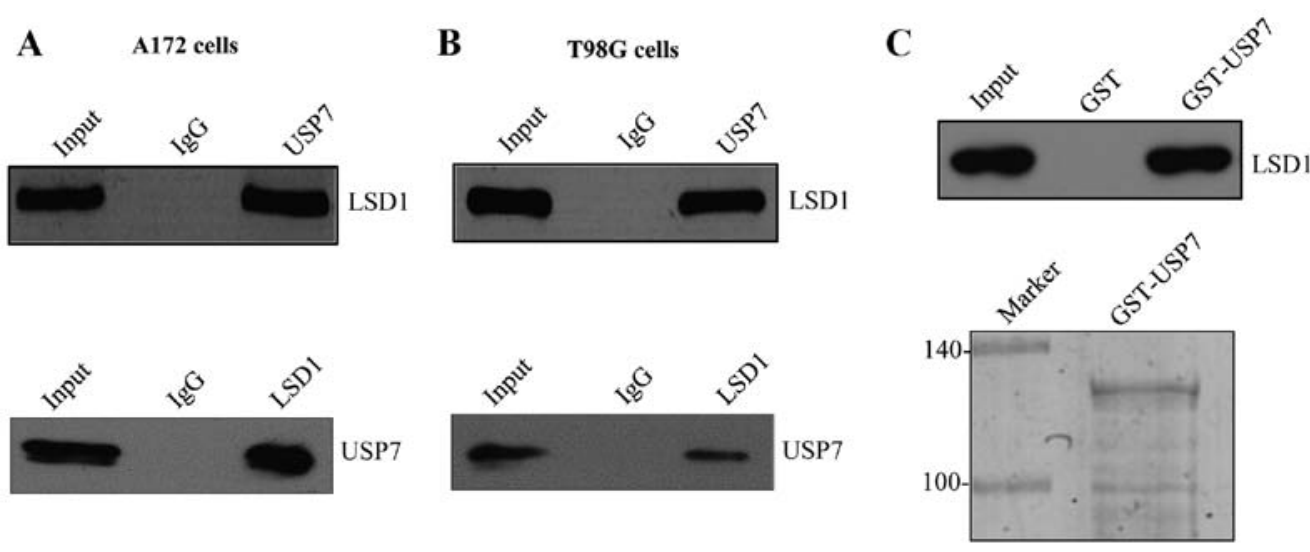

Figure 1. LSD1 is identified as a USP7-interacting protein. (A) Co-immunoprecipitation assay (co-IP) analysis was used to detect the association between USP7 and LSD1. A172 cell lysates were immunoprecipitated with USP7; normal immunoglobin G (IgG) was used as the negative control and whole lysates as the positive control. The immunocomplexes were then immunoblotted using LSD1, reciprocally. (B) T98G cell lysates were further used to detect the association between USP7 and LSD1. (C) Bacterially expressed GST-USP7 and transcribed LSD1 were used to perform a GST pull-down assay. LSD1, lysine-specific demethylase 1; USP7, ubiquitin specific peptidase 7.

EdU cell proliferation assay. Cells were seeded onto 24-well plates for $24 \mathrm{~h}$ and transfected with the indicated siRNAs or plasmids using Lipofectamine 2000 (Invitrogen Life Technologies). Cell proliferation ELISA EdU kits (Roche Applied Science, Indianapolis, IN, USA) were used according to the manufacturer's instructions. Forty-eight hours after transfection, 5-ethynyl-2'-deoxyuridine (EdU) was added to the medium for an additional $4 \mathrm{~h}$ at $37^{\circ} \mathrm{C}$, and then the wells were washed 3 times with washing solution $(200 \mu \mathrm{l} /$ well). Four percent formaldehyde was added to the cells for $30 \mathrm{~min}$, and $2 \mathrm{mg} / \mathrm{ml}$ glycine was incubated for $5 \mathrm{~min}$. Finally, 4',6-diamidino-2-phenylindole (DAPI) was used to stain the nuclei. The experiments were performed in triplicate.

Transwell assay. A Transwell chamber was precoated with $6 \mu \mathrm{l}$ Matrigel at $4^{\circ} \mathrm{C}$ overnight and $5 \times 10^{4}$ cells were seeded into $500 \mu \mathrm{l}$ serum-free DMEM on the upper chamber. DMEM supplemented with $10 \%$ FBS was added to the lower chamber. After being allowed to invade for $10 \mathrm{~h}$, the invading cells on the lower surface were fixed with $70 \%$ ethanol followed by crystal violet staining, while the remaining cells on the upper chamber were removed using cotton swabs. The number of invaded cells was calculated under a microscope in four random fields of vision.

Statistical analysis. SPSS software 17.0 (SPSS Inc., Chicago, IL, USA) was used for statistical analysis. The significance between groups was analyzed using one-way analysis of variance (ANOVA) and Student's t-tests. A Kaplan-Meier plot and univariate Cox regression analysis were used to analyze the glioma patient morbidity. Log-rank analysis was used to test the differences between groups. The means \pm standard deviation was calculated for all experiments. $\mathrm{P}<0.05$ was considered to be statistically significance; ${ }^{*} \mathrm{P}<0.05,{ }^{* *} \mathrm{P}<0.01$ are indicated in the figures and legends.

\section{Results}

LSD1 is identified as a USP7-interacting protein. In order to investigate the potential substrates of USP7 in glioblastoma, we first used co-IP to identify the USP7-interacting proteins in A172 cells, as shown in Fig. 1A, firstly, by immunoprecipitation with antibodies against USP7, and then followed by western blotting against LSD1 antibodies. The data indicated that USP7 was associated with LSD1 in vivo, but not with the IgG control. Reciprocal immunoprecipitation with anti-LSD1 and immunoblotting with anti-USP7 also indicated this fact. This interaction was also confirmed with endogenous proteins in the T98G cells (Fig. 1B) Notably, this interaction was specific, since USP7 did not associate with another lysine-specific demethylase 2 (LSD2) (data not shown).

To investigate whether USP7 interacts with LSD1 directly, a GST pull-down experiment was performed. Glutathione S-transferase fusion protein (GST-ZIP) was immobilized on glutathione Sepharose 4B beads, and subsequently incubated with in vitro transcribed/translated FLAG-tagged LSD1, as shown in Fig. 1C. The GST-USP7 fusion protein was found to bind with LSD1 directly in vitro. Collectively, these experiments supported our observation that USP7 is physically associated with LSD1 in vivo and in vitro.

USP7 inhibits LSD1 ubiquitination and stabilizes LSD1 in vivo. Since USP7 was shown to deubiquitinate and stabilize p53, MDM2 and SIRT1, it was also feasible that USP7 may regulate LSD1 function via its deubiquitinase activity. Firstly, A172 cells as well as T98G cells were infected with a control siRNA, siUSP7\#1, or siUSP7\#2 and the knockdown efficiency was detected. As shown in Fig. 2A, the USP7 mRNA level was significantly reduced when cells were infected with siUSP7\#1 or siUSP7\#2 as compared with the level in the control siRNA group. Concomitantly, the protein level, as detected by western blotting revealed that USP7 expression was also greatly reduced in the siRNA groups than that noted in the control group and siUSP7\#2 was more effective and used for further experiments. Furthermore, after USP7 was knocked down, we investigated the expression of LSD1 and as expected, although the mRNA level was not changed (Fig. 2B, left panels), the protein level of LSD1 was dramatically reduced (Fig. 2B, right panels). This indicated that USP7 may influence LSD1 through post-transcription modification. Consistently, we 

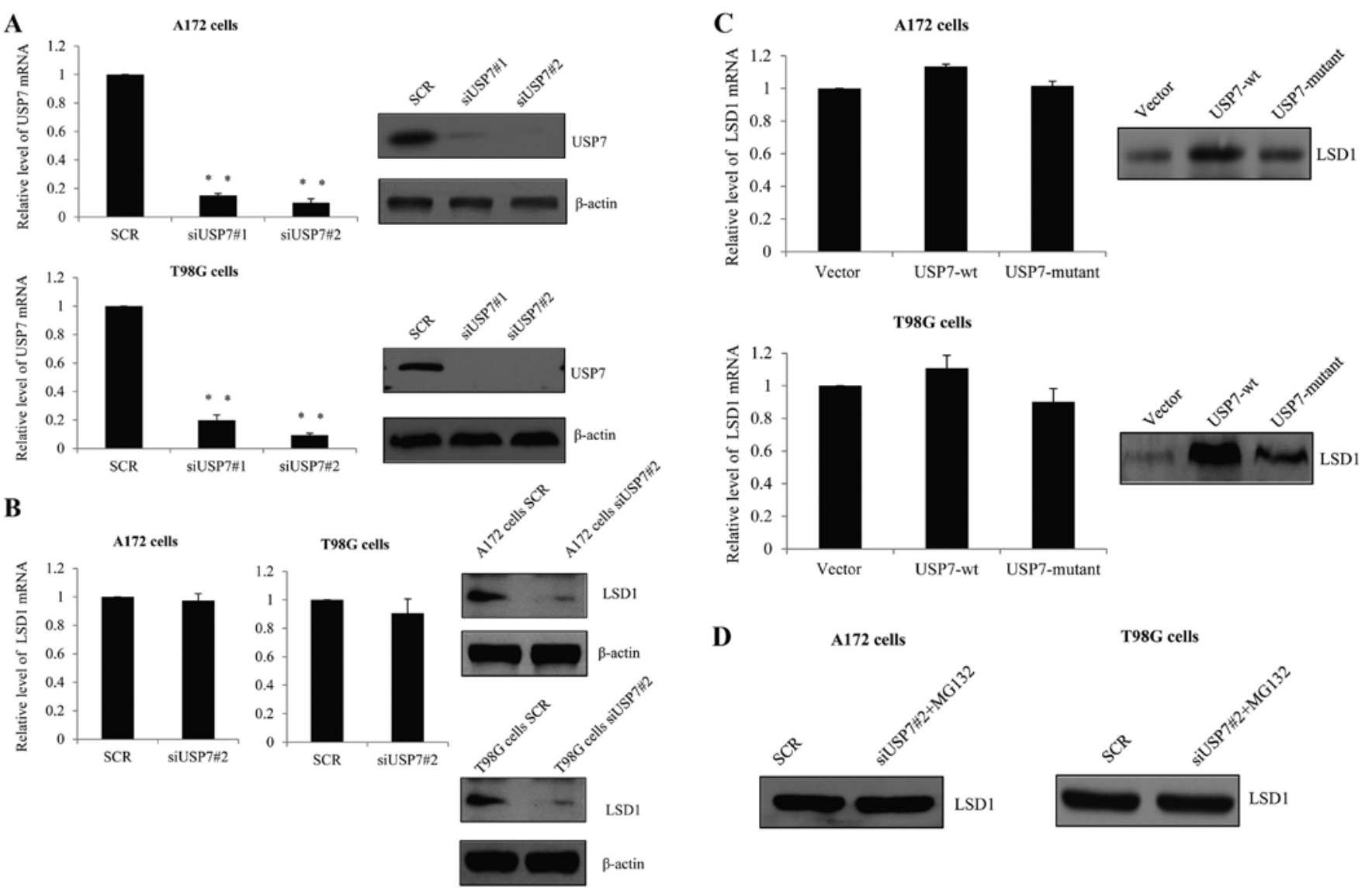

D
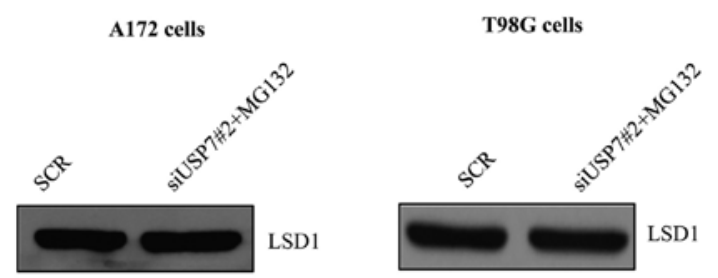

Figure 2. USP7 inhibits LSD1 ubiquitination and stabilizes LSD1 in vivo. (A) A172 and T98G cells were transfected with control siRNA, siUSP7\#1 or siUSP7\#2. The knockdown efficiency of USP7 was confirmed by quantitative real-time PCR (left panels) and western blotting (right panels). ${ }^{* *} \mathrm{P}<0.01$. (B) A172 and T98G cells were transfected with control siRNA or siUSP7\#1 and the mRNA and protein levels of LSD1 were detected. (C) A172 and T98G cells were transfected with vector, wt-USP7 or USP7 mutant and the mRNA and the protein levels of LSD1 were detected. (D) USP7 was knocked down in the A172 and T98G cells, and the cells were incubation with MG132 before harvesting. Western blotting was used to measure LSD1 protein expression. USP7, ubiquitin specific peptidase 7; LSD1, lysine-specific demethylase 1.

overexpressed wild-type USP7 plasmid or catalytically inactive USP7 (USP7/C223A) mutant plasmid in the A172 and $\mathrm{T} 98 \mathrm{G}$ cells and it was revealed that the protein level of LSD1 only increased in the wt-USP7-transfected groups, but not in the catalytically inactive USP7 (USP7/C223A) mutant group (Fig. 2C), where there was no marked change in the mRNA level.

To determine the effect of USP7 on LSD1 protein ubiquitination, USP7 was knocked down by siRNA in the A172 and T98G cells, and the cells were harvested after MG132 (a proteasome-specific inhibitor) treatment. We found that MG132 was successful in rescuing the LSD1 protein but not mRNA from the degradation in the USP7-knockdown cells (Fig. 2D). Based on the aforementioned experiments, we demonstrated that USP7 could stabilize LSD1 possibly based on its deubiquitinating activity.

LSD1 plays a critical role in the proliferation and invasion of glioblastoma cells. As reported, LSD1 is overexpressed in glioblastoma (9), but the function of LSD1 requires further exploration. Thus, to investigate the function, two specific siRNAs targeting LSD1 were successfully developed and transfected into the A172 and T98G cells. The knockdown efficiency was nearly 90\%, and as shown in Fig. 3A, siLSD1\#2 was more effective and was used for further experimentation.
As shown in Fig. 3B, the proliferation of the A172 and T98G cells as determined by MTT assay was significantly suppressed after treatment with siLSD1 compared with the control group of cells. The result were corroborated using the EdU proliferation assay (Fig. 3C). In the cell cycle distribution assay, knockdown of LSD1 caused G0/G1 arrest compared with the control group (Fig. 3D). In the Transwell assay, there were less cells that crossed the membranes in the LSD1-knockdown group than that noted in the control group (Fig. 3E). The aforementioned results illustrated that the downregulation of LSD1 resulted in the inhibition of glioblastoma cell proliferation and invasion.

USP7 promotes glioblastoma cell tumorigenesis by regulation of LSD1. In order to evaluate the effect of USP7 on glioblastoma tumorigenesis, USP7 was knocked down in the A172 (Fig. 4A, left panel) and T98G cells (Fig. 4A, right panel) An MTT assay was performed. When compared with the control siRNA groups, the knockdown of USP7 effectively inhibited the cell growth rate. Similarly, EdU cell proliferation assay was performed in the aforementioned two cell lines (Fig. 4B), and also demonstrated that knockdown of USP7 reduced their proliferative ability.

The cell cycle distribution by FCM confirmed that in the A172 and T98G cells, compared with the control cells, knockdown of USP7 caused G0/G1 arrest (Fig. 4C), and 
A
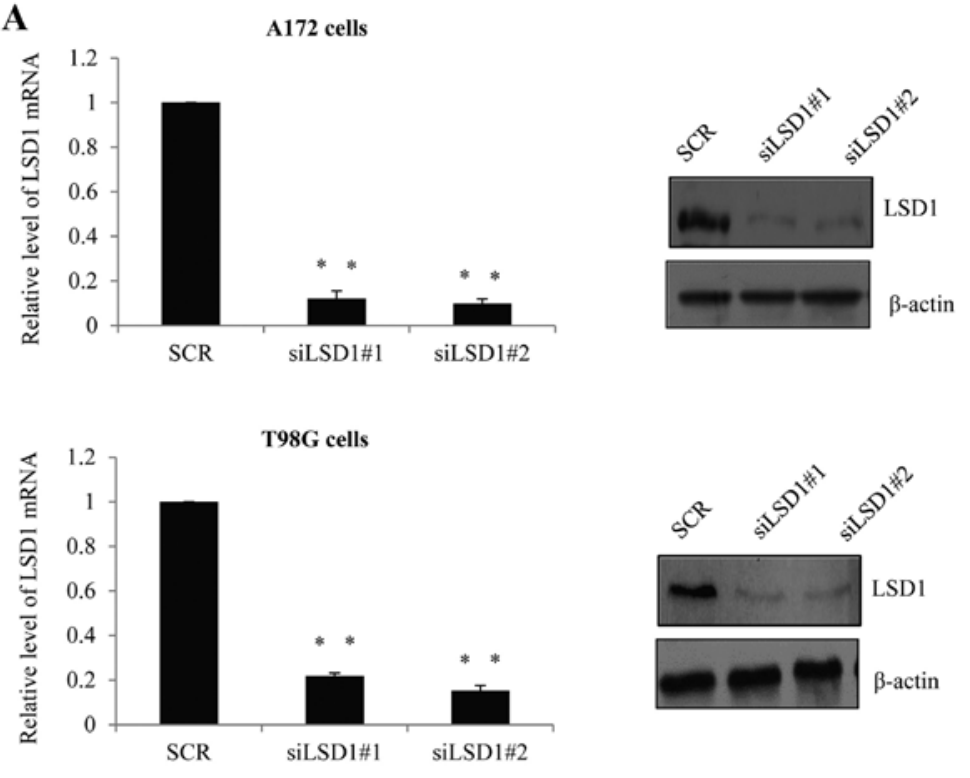

B

A172 cells
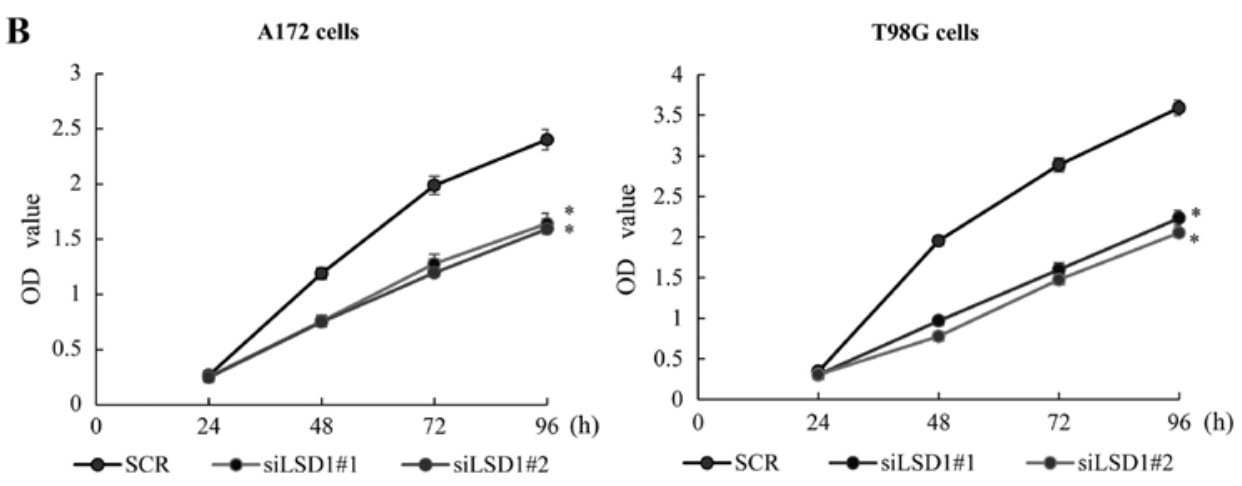

Figure 3. LSD1 plays a critical role in the proliferation and invasion of glioblastoma cells. (A) A172 and T98G cells were transfected with control siRNA, siLSD1\#1 or siLSD1\#2 and the knockdown efficiency of LSD1 was confirmed by quantitative real-time PCR (left panels) and western blotting (right panels). ${ }^{* *} \mathrm{P}<0.01$. (B) An MTT assay was used to measure the A172 and T98G cell proliferation. An equal amount of cells was transfected with siLSD1 or with the relative control siRNA group (control) and cells were seeded into replicate plates and counted every $12 \mathrm{~h}$. Data are represented as the means \pm SD of 3 independent experiments. ${ }^{*} \mathrm{P}<0.05$. LSD1, lysine-specific demethylase 1.

inhibited cell growth. These observations strongly suggested that USP7 may play a vital role in promoting GBM cell proliferation in vitro. We examined the role of LSD1 in the USP7-mediated GBM cell tumorigenesis using the MTT assay. The inhibiton of cell proliferation resulting from knockdown of USP7 was partially affected by LSD1 overepression in the A172 and T98G cells (Fig. 4D). Furthermore, upon treatment with siUSP7, EdU proliferation assay indicated that the inhibition of proliferation by USP7 knockdown in the A172 and T98G cells was markedly augmented by LSD1 overexpression (Fig. 4E). Collectively, these results indicated that LSD1 had an obviously impact on the ability of USP7 to promote cell proliferation.

USP7 affects glioblastoma cell invasiveness through stabilization of LSDI. The Transwell assay was used to investigate the impact of USP7 expression on GBM cell invasion. An equal amount of A172 control or A172 siUSP7 cells were placed into the Transwell chamber and as shown in Fig. 5A, the knockdown of USP7 cells exhibited poor invasive ability $(\mathrm{P}<0.05)$. A similar tendency was also observed between the T98G-siUSP7 and T98G-control cells (Fig. 5B). These data indicated that USP7 increased the invasive ability of GBM cells.

To confirm whether LSD1 is involved in USP7-triggered glioblastoma cell invasiveness, LSD1 was overexpressed in the siUSP7\#2-transfected A172 cells, and a Transwell assay was performed. The effect of USP7 was reduced, as shown in Fig. 5C. Furthermore, we created a USP7-knockdown T98G cell line, and a similar tendency was also observed with ectopic expression of LSD1 (Fig. 5D). These results revealed that knockdown of USP7 inhibited glioblastoma cell invasiveness through the regulation of LSD1.

Suppression of the p53 signaling pathway is involved in the USP7-LSD1 regulated glioblastoma cell tumorigenesis and metastasis. To investigate the potential downstream effectors which are regulated by USP7 and LSD1, we next examined several signaling pathways that contributed to cancer cell proliferation and invasion, such as the p53, the AKT and the Bcl2 apoptosis pathways. As shown in Fig. 6A, in the LSD1knockdown A172 and T98G cells, the mRNA expression of p53 increased while the mRNA expression of Akt1 and 

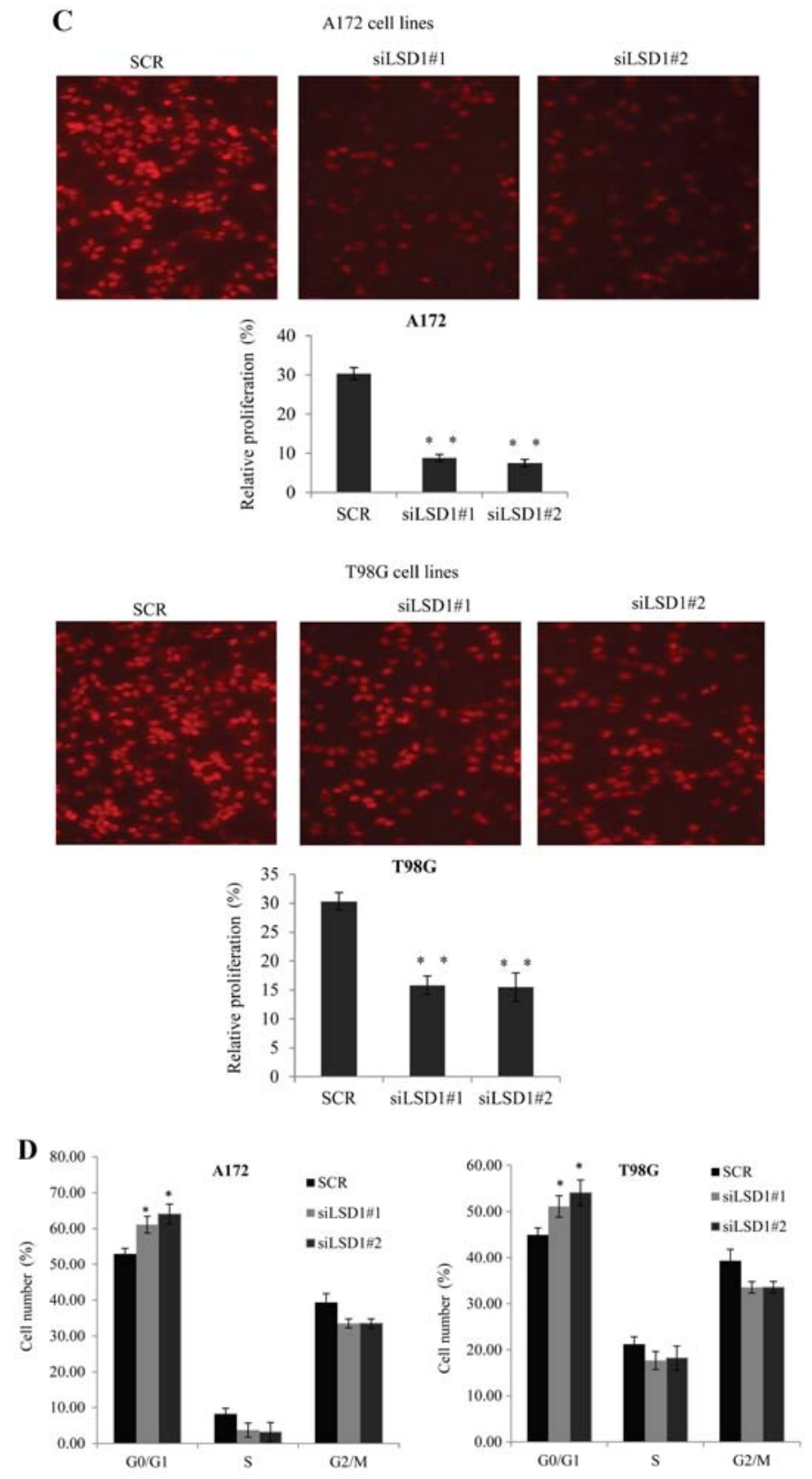

$\mathbf{E}$ $\mathrm{SCR}$ siLSDI\#1 siLSD1\#2
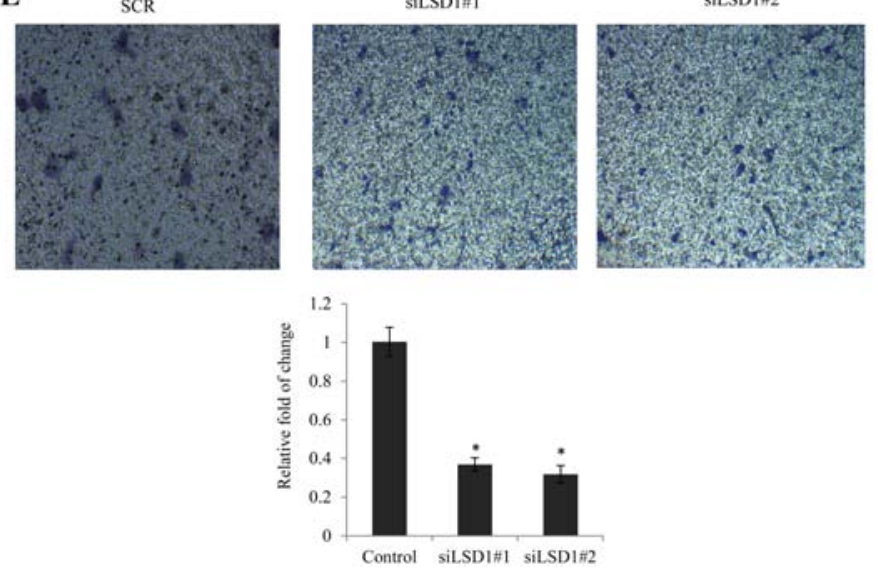

Figure 3. Continued. LSD1 plays a critical role in the proliferation and invasion of glioblastoma cells. (C) EdU cell proliferation assay was performed in the A172 and T98G cells transfected with siLSD1 or a vector as a control. Representative images are shown for each group. (D) Cell cycle distribution was performed by flow cytometry (FCM) in the siLSD1-transfected A172 and T98G cells. The percentage of cells in the G0/G1, S, and G2/M phases are shown as indicated. (E) A172 cells were transfected with control siRNA or siLSD1. Forty-eight hours after transfection, a Transwell assay was performed, and the invaded cells were stained and counted. Statistical analysis is presented as a fold of change over the control. Bars indicate the mean \pm SD of three independent experiments. ${ }^{*} \mathrm{P}<0.05 ;{ }^{* *} \mathrm{P}<0.01$. LSD1, lysine-specific demethylase 1. 
A

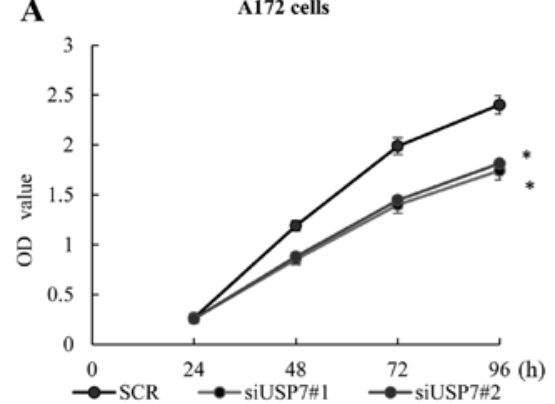

B

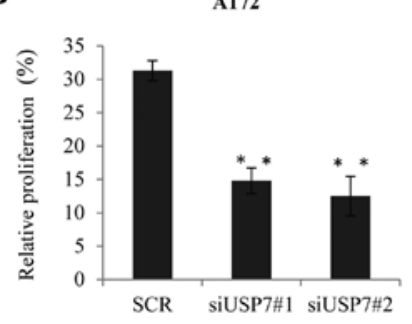

C

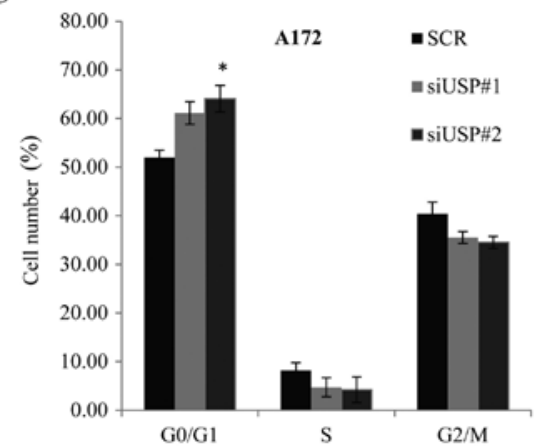

T98G cells

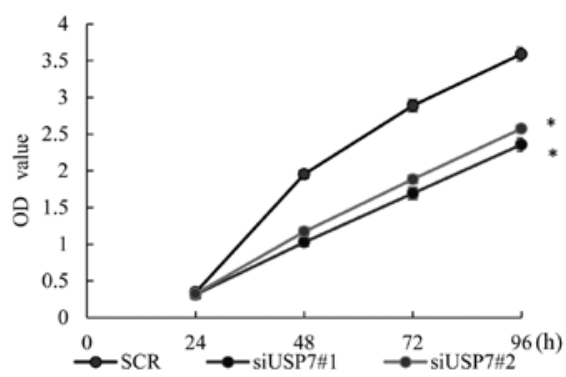

T98G
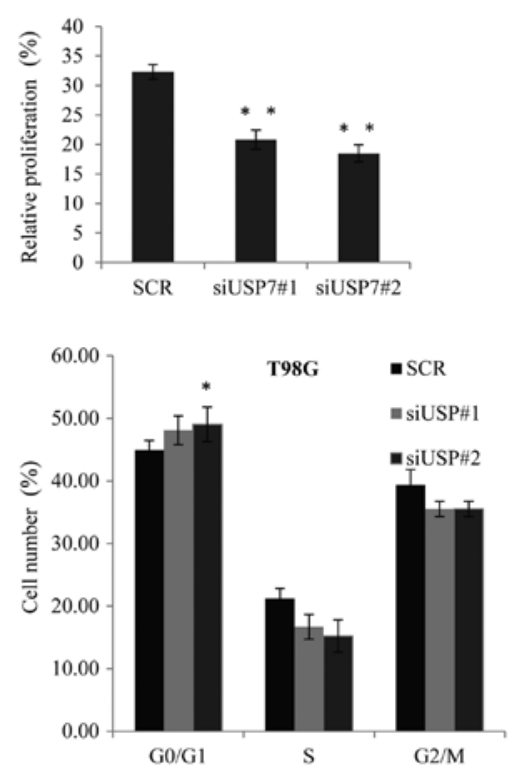

D
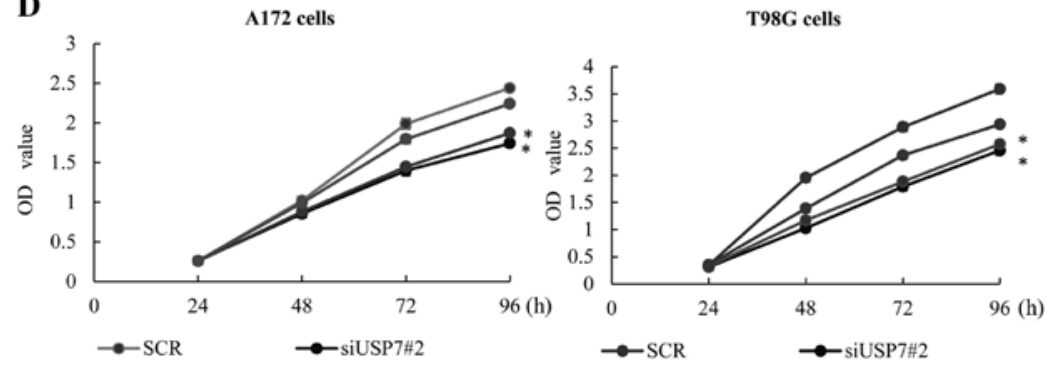

$\rightarrow-$ siUSP7\#2+vector $\rightarrow-$ siUSP7\#2+LSD1

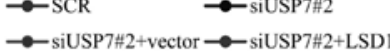

E

A172
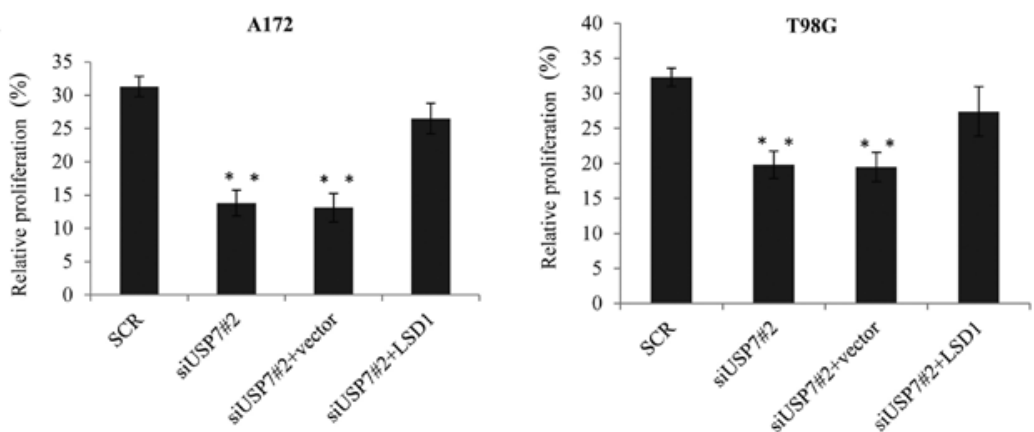

Figure 4. USP7 promotes glioblastoma cell tumorigenesis by regulation of LSD1. (A) An MTT assay was used to measure the A172 and T98G cell proliferation. An equal amount of cells was transfected with siUSP7 or with the relative control siRNA group (control). (B) EdU cell proliferation assay was performed in the A172 and T98G cells transfected with siUSP7 or the control siRNA. (C) Cell cycle distribution was performed by flow cytometry (FCM) in the siUSP7 transfected A172 and T98G cells. The percentage of cells in the G0/G1, S, and G2/M phases are shown as indicated. (D) An MTT assay was performed in the siUSP7\#2-transfected A172 cells, followed by transient transfection with a vector or LSD1 plasmid. A similar experiment was also performed with the T98G cells. (E) EdU cell proliferation assay was carried out in the siUSP7\#2-transfected A172 cells, followed by transient transfection with a vector or LSD1 plasmid. A similar experiment was also performed with the T98G cells. " $\mathrm{P}<0.05 ;{ }^{* *} \mathrm{P}<0.01$. USP7, ubiquitin specific peptidase 7; LSD1, lysine-specific demethylase 1. 
A

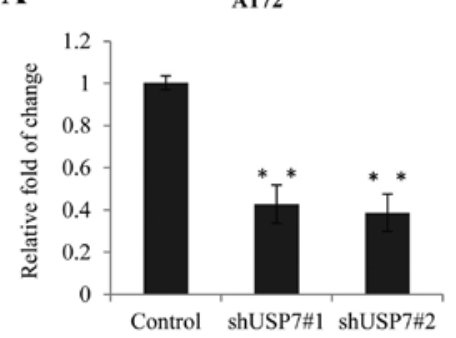

C

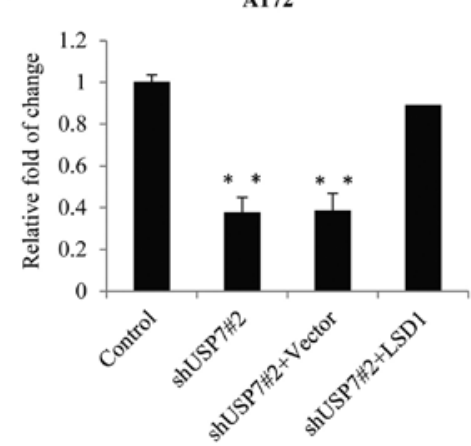

B

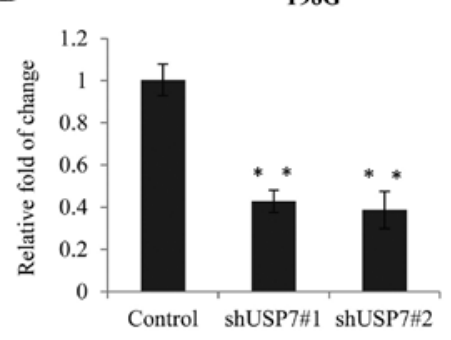

D

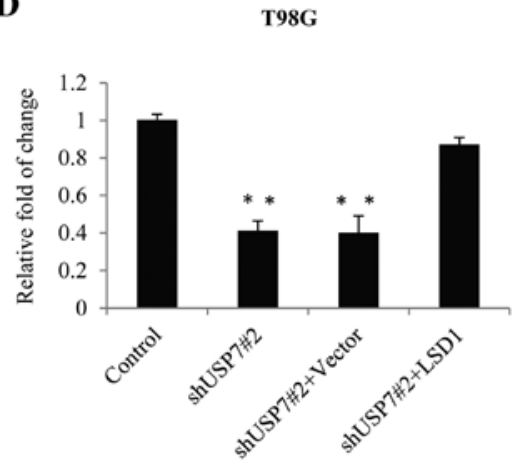

Figure 5. USP7 affects glioblastoma cell invasiveness through stabilizion of LSD1. (A) A Transwell assay was performed in the A172 cells transfected with the control siRNA or siUSP7. (B) The T98G cells were transfected with the control siRNA or siUSP7, and similarly, a Transwell assay was performed. (C) A Transwell assay was performed in the siUSP7\#2-transfected A172 cells, followed by transient transfection with a vector or LSD1 plasmid. (D) A similar experiment was performed with the T98G cells. ${ }^{* *} \mathrm{P}<0.01$. USP7, ubiquitin specific peptidase 7; LSD1, lysine-specific demethylase 1.
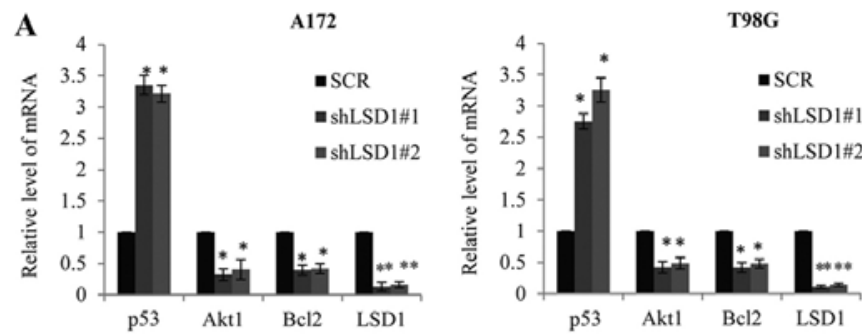

B
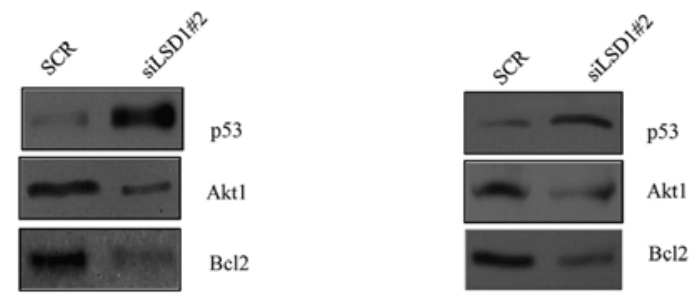

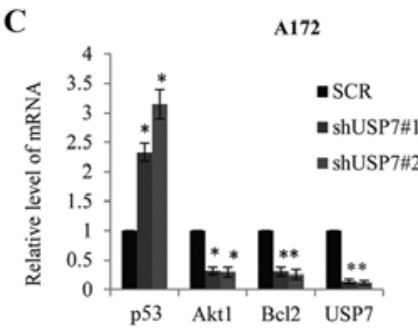

D

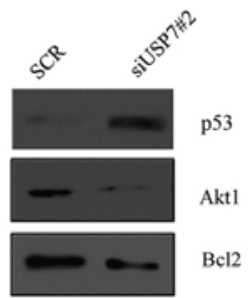

T98G
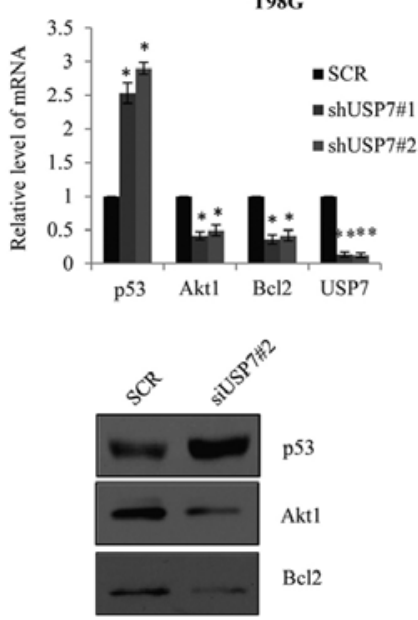

Figure 6. Suppression of the p53 signaling pathway is involved in the USP7-LSD1 regulated glioblastoma cell tumorigenesis and metastasis. (A) When LSD1 was knocked down in the A172 and T98G cells, the mRNA expression of p53, Akt1 and Bcl2 was assessed. ${ }^{*} \mathrm{P}<0.05,{ }^{* *} \mathrm{P}<0.01$. (B) In the LSD1-knockdown A172 and T98G cells, the protein expression of p53 Akt1 and Bcl2 was assessed and $\beta$-catenin was used as a control. In the A172-siUSP7 cells and T98GsiUSP7 cells, the (C) mRNA and (D) protein expression of p53, Akt1 and Bcl2 were detected. USP7, ubiquitin specific peptidase 7; LSD1, lysine-specific demethylase 1 .

Bcl2 were decreased when compared to the control group cells. Using western blotting (Fig. 6B), we confirmed that the protein expression of p53 was higher in the A172-siLSD1 or the T98G-siLSD1 cells compared with the vector, while the expression of Akt1 and Bcl2 presented the opposite tendency. Consistently, in USP7 knockdown in the A172 cells or T98G cells, the mRNA (Fig. 6C) and protein expression (Fig. 6D) of p53, Akt1 and $\mathrm{Bcl} 2$ revealed similar results. Considering the transcription repression function of LSD1, it is reasonable to make the hypothesis that LSD1 could suppress p53 directly. These results suggested that USP7 and p53 are upstream and downstream regulators of LSD1-mediated proliferation and invasion. and positively correlated with poor GBM prognosis. Using 
A

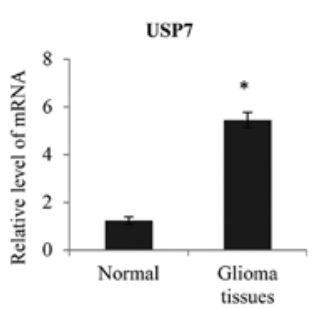

B

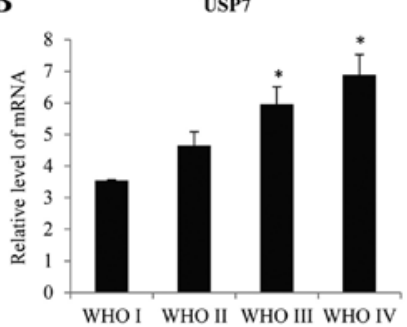

C

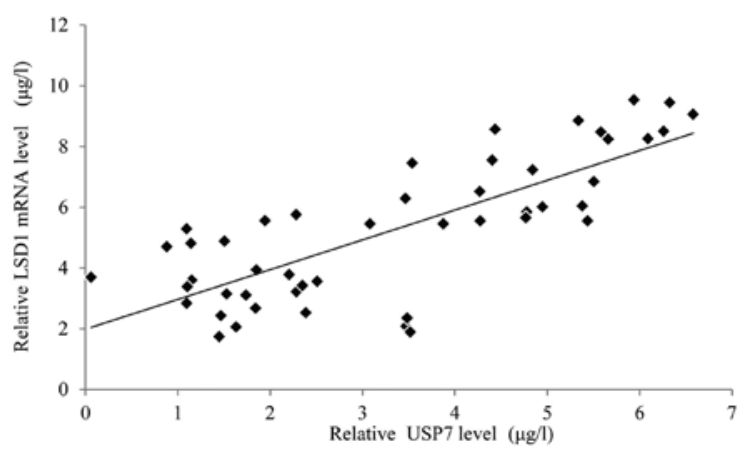

D

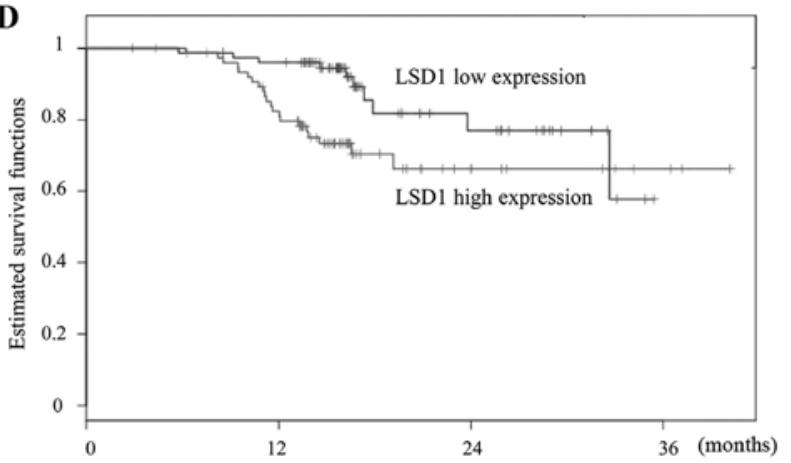

E

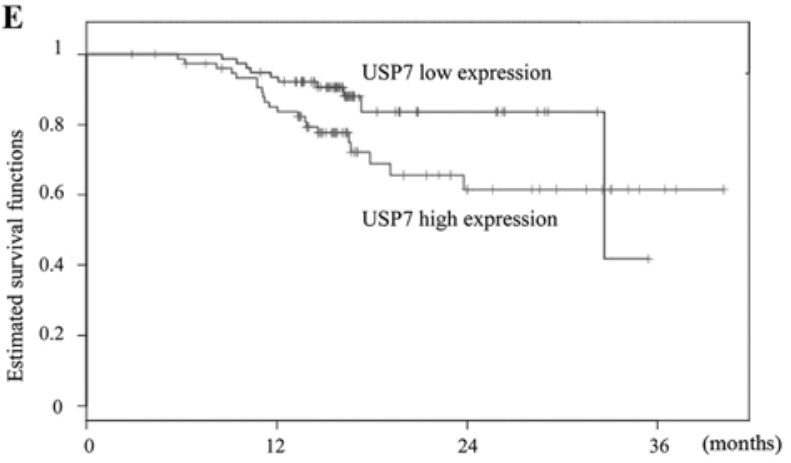

Figure 7. USP7 and LSD1 are frequently upregulated in glioblastoma multiforme (GBM) and positively correlated with poor GBM prognosis. (A) LSD1 and USP7 mRNA expression in non-brain tumor and 150 glioma tissues. ${ }^{*} \mathrm{P}<0.05$. (B) Relative mRNA level of LSD1 and USP7 expression in glioma of different World Health Organization (WHO) grades was determined by qRTPCR. GAPDH was used for normalization. " $\mathrm{P}<0.05$. (C) Correlation between USP7 and LSD1 in the WHO grade IV cancer samples from 48 patients. (D) Kaplan-Meier curves were used to compare the overall survival rates of the glioma patients according to LSD1 expression. (E) Kaplan-Meier curves were drawn based on USP7 expression. USP7, ubiquitin specific peptidase 7; LSD1, lysine-specific demethylase 1.
Table I. Mean values of LSD1 mRNA expression in the clinical glioma samples and normal control tissues, and comparison with clinicopathological variables.

\begin{tabular}{lrcc}
\hline Variables & $\mathrm{n}$ & $\begin{array}{c}\text { LSD1 expression } \\
(\text { mean } \pm \text { SD })\end{array}$ & P-value \\
\hline $\begin{array}{l}\text { Tissue type } \\
\text { Glioma }\end{array}$ & 150 & $6.973 \pm 0.454$ & $<0.05$ \\
$\quad$ Normal control & 10 & $1.342 \pm 0.239$ & \\
Gender & & & $>0.05$ \\
Male & 86 & $6.731 \pm 0.872$ & \\
Female & 64 & $6.422 \pm 0.764$ & \\
Age (years) & & & $>0.05$ \\
$<60$ & 84 & $6.453 \pm 0.784$ & \\
$\geq 60$ & 66 & $6.891 \pm 0.545$ & \\
KPS & & & \\
$<80$ & 73 & $6.584 \pm 0.713$ & \\
$\geq 80$ & 77 & $6.136 \pm 0.376$ & \\
WHO grade & & & \\
I & 28 & $3.741 \pm 0.131$ & \\
II & 29 & $5.349 \pm 0.394$ & \\
III & 45 & $7.032 \pm 0.579$ & \\
IV & 48 & $8.712 \pm 0.732$ & \\
\hline
\end{tabular}

LSD1, lysine-specific demethylase 1; WHO, World Health Organization;.

Table II. Mean values of USP7 mRNA expression in clinical glioma samples and comparison with clinicopathological variables.

\begin{tabular}{|c|c|c|c|}
\hline Variables & $\mathrm{n}$ & $\begin{array}{l}\text { USP7 expression } \\
\quad(\text { mean } \pm \text { SD })\end{array}$ & P-value \\
\hline Tissue type & & & $<0.05$ \\
\hline Glioma & 150 & $5.453 \pm 0.324$ & \\
\hline Normal control & 10 & $1.231 \pm 0.159$ & \\
\hline Gender & & & $>0.05$ \\
\hline Male & 86 & $5.671 \pm 0.592$ & \\
\hline Female & 64 & $5.223 \pm 0.743$ & \\
\hline Age (years) & & & $>0.05$ \\
\hline$<50$ & 84 & $5.243 \pm 0.954$ & \\
\hline$\geq 50$ & 66 & $5.784 \pm 0.375$ & \\
\hline KPS & & & $<0.05$ \\
\hline$<80$ & 73 & $5.754 \pm 0.518$ & \\
\hline$\geq 80$ & 77 & $5.232 \pm 0.459$ & \\
\hline WHO grade & & & $<0.05$ \\
\hline $\mathrm{I}$ & 28 & $3.543 \pm 0.231$ & \\
\hline II & 29 & $4.649 \pm 0.434$ & \\
\hline III & 45 & $5.958 \pm 0.549$ & \\
\hline IV & 48 & $6.894 \pm 0.632$ & \\
\hline
\end{tabular}

USP7, ubiquitin specific peptidase 7; WHO, World Health Organization. 
quantitative real-time PCR (qRT-PCR), we determined the LSD1 and USP7 mRNA expression, normalized to GAPDH. As shown in Fig. 7A, we found that there was no obvious increase in the LSD1 and USP7 expression in 150 glioma tissues compared with non-brain tumor tissues $(\mathrm{P}<0.05)$. There were 57 low-grade (WHO grades I and II) and 103 high-grade tumors (WHO grades III and IV) (Tables I and II). Based on the WHO grade, we found that LSD1 and USP7 mRNA expression increased notably with the advancement of WHO grade I to IV (Fig. 7B, $\mathrm{P}<0.05$ ). Meanwhile, there was a significant positive correlation between the LSD1 expression and USP7 expression in the high-grade GBC tissues (WHO grade IV glioblastoma) (Fig. 7C, $\mathrm{R}=0.06, \mathrm{P}<0.05$ ). Furthermore, according to the details of the follow-up, patients with a higher LSD1 expression had a worse overall survival after surgery (Fig. 7D, $\mathrm{P}<0.05$ ), and a similar tendency was also observed in patients with a higher USP7 expression (Fig. 7E, P $<0.05$ ). These results suggested that LSD1 and USP7 are upregulated in GBC tissues and associated with poor prognosis.

\section{Discussion}

A number of studies have reported that USP7 may regulate several polyubiquitinated substrates by its deubiquitinating activity. As reported (20), destabilization of LSD1 occurs via the ubiquitin-proteasome pathway by an E3 ubiquitin ligase, Jade-2 during neurogenesis, but the stabilization of LSD1 is unclear.

In the present study, we first discovered LSD1 as the new substrate of USP7 from a molecular level. The evidence is as follows. Firstly, we identified LSD1 as an associated protein by immunoprecipitation with USP7 but no other DUB proteins, and GST pull-down assays confirmed that LSD1 could interact with USP7 directly. Notably, overexpression of USP7 increased the protein level of LSD1 but not the mRNA level. The mechanism involved is that USP7 could protect ubiquitination and the proteasomal degradation of LSD1. As reported, expression of USP7 in gliomas was correlated with disease progression and patient survival time (21), but the mechanism involved is still unclear. In order to further understand the role of USP7 and LSD1 in glioblastoma progression, we investigated the expression of USP7 and LSD1 in 150 cases of human glioma and normal brain tissues and our data demonstrated that USP7 and LSD1 mRNA expression was higher compared with the normal brain tissues with an increasing trend from grade I to grade IV glioma according to WHO classification. Furthermore, the expression of LSD1 was positively correlated with USP7 expression in human glioma. These results suggested that the stabilization of LSD1 by USP7 may participate in glioma progression. We defined the function of USP7 and LSD1 in cell proliferation and invasion using GBM cell lines A172 and T98G, as the ectopic expression of LSD1 could partly induce the tendency by USP7 knockdown in the proliferation and invasion of GBM cells. As reported, LSD1 mediated various epigenetic gene expression regulating effects, such as p53 (22). In this study, we confirmed that p53 is a key downstream transcription factor that mediates the action of USP7 and LSD1. The aforementioned experiments further confirmed that the regulation of LSD1 by USP7 plays an important role in GBM and may function as a therapeutic target in GBM.

\section{Acknowledgements}

This study was supported by HuNan Provincial Innovation Foundation for Postgraduate (No. CX2015B062).

\section{References}

1. Lan F, Nottke AC and Shi Y: Mechanisms involved in the regulation of histone lysine demethylases. Curr Opin Cell Biol 20: 316-325, 2008.

2. Prusevich P, Kalin JH, Ming SA, Basso M, Givens J, Li X, Hu J, Taylor MS, Cieniewicz AM, Hsiao PY, et al: A selective phenelzine analogue inhibitor of histone demethylase LSD1. ACS Chem Biol 9: 1284-1293, 2014.

3. Amente S, Milazzo G, Sorrentino MC, Ambrosio S, Di Palo G, Lania L, Perini G and Majello B: Lysine-specific demethylase (LSD1/KDM1A) and MYCN cooperatively repress tumor suppressor genes in neuroblastoma. Oncotarget 6: 14572-14583, 2015.

4. Lv T, Yuan D, Miao X, Lv Y, Zhan P, Shen X and Song Y: Over-expression of LSD1 promotes proliferation, migration and invasion in non-small cell lung cancer. PLoS One 7: e35065, 2012.

5. Huang Z, Li S, Song W, Li X, Li Q, Zhang Z, Han Y, Zhang X, Miao S, Du R, et al: Lysine-specific demethylase 1 (LSD1/KDM1A) contributes to colorectal tumorigenesis via activation of the Wnt/ $\beta$-catenin pathway by down-regulating Dickkopf-1 (DKK1) [corrected]. PLoS One 8: e70077, 2013.

6. Ketscher A, Jilg CA, Willmann D, Hummel B, Imhof A, Rüsseler V, Hölz S, Metzger E, Müller JM and Schüle R: LSD1 controls metastasis of androgen-independent prostate cancer cells through PXN and LPAR6. Oncogenesis 3: e120, 2014.

7. Wang Y, Zhang H, Chen Y, Sun Y, Yang F, Yu W, Liang J, Sun L, Yang X, Shi L, et al: LSD1 is a subunit of the NuRD complex and targets the metastasis programs in breast cancer. Cell 138: 660-672, 2009

8. Si W, Huang W, Zheng Y, Yang Y, Liu X, Shan L, Zhou X, Wang Y, Su D, Gao J, et al: Dysfunction of the reciprocal feedback loop between GATA3- and ZEB2-nucleated repression programs contributes to breast cancer metastasis. Cancer Cell 27: 822-836, 2015.

9. Singh MM, Manton CA, Bhat KP, Tsai WW, Aldape K, Barton MC and Chandra J: Inhibition of LSD1 sensitizes glioblastoma cells to histone deacetylase inhibitors. Neuro Oncol 13: 894-903, 2011.

10. Li M, Chen D, Shiloh A, Luo J, Nikolaev AY, Qin J and Gu W: Deubiquitination of p53 by HAUSP is an important pathway for p53 stabilization. Nature 416: 648-653, 2002.

11. Brooks CL,Li M,Hu M,Shi Y and Gu W: The p53-Mdm2-HAUSP complex is involved in 553 stabilization by HAUSP. Oncogene 26: 7262-7266, 2007.

12. Saridakis V, Sheng Y, Sarkari F, Holowaty MN, Shire K, Nguyen T, Zhang RG, Liao J, Lee W, Edwards AM, et al: Structure of the p53 binding domain of HAUSP/USP7 bound to Epstein-Barr nuclear antigen 1 implications for EBV-mediated immortalization. Mol Cell 18: 25-36, 2005.

13. Song MS, Salmena L, Carracedo A, Egia A, Lo-Coco F, Teruya-Feldstein $\mathrm{J}$ and Pandolfi PP: The deubiquitinylation and localization of PTEN are regulated by a HAUSP-PML network. Nature 455: 813-817, 2008.

14. Maertens GN, El Messaoudi-Aubert S, Elderkin S, Hiom K and Peters G: Ubiquitin-specific proteases 7 and 11 modulate Polycomb regulation of the INK4a tumour suppressor. EMBO J 29: 2553-2565, 2010.

15. van der Horst A, de Vries-Smits AM, Brenkman AB, van Triest MH, van den Broek N, Colland F, Maurice MM and Burgering BM: FOXO4 transcriptional activity is regulated by monoubiquitination and USP7/HAUSP. Nat Cell Biol 8: 1064-1073, 2006.

16. Huang Z, Wu Q, Guryanova OA, Cheng L, Shou W, Rich JN and Bao S: Deubiquitylase HAUSP stabilizes REST and promotes maintenance of neural progenitor cells. Nat Cell Biol 13: 142-152, 2011.

17. Louis DN, Ohgaki H, Wiestler OD, Cavenee WK, Burger PC, Jouvet A, Scheithauer BW and Kleihues P: The 2007 WHO classification of tumours of the central nervous system. Acta Neuropathol 114: 97-109, 2007. 
18. Erpolat OP, Akmansu M, Goksel F, Bora H, Yaman E and Büyükberber S: Outcome of newly diagnosed glioblastoma patients treated by radiotherapy plus concomitant and adjuvant temozolomide: A long-term analysis. Tumori 95: 191-197, 2009.

19. Stupp R, Mason WP, van den Bent MJ, Weller M, Fisher B, Taphoorn MJ, Belanger $\mathrm{K}$, Brandes AA, Marosi C, Bogdahn U, et al; European Organisation for Research and Treatment of Cancer Brain Tumor and Radiotherapy Groups; National Cancer Institute of Canada Clinical Trials Group: Radiotherapy plus concomitant and adjuvant temozolomide for glioblastoma. N Engl J Med 352: 987-996, 2005.
20. Han X, Gui B, Xiong C, Zhao L, Liang J, Sun L, Yang X, Yu W, Si W, Yan R, et al: Destabilizing LSD1 by Jade-2 promotes neurogenesis: An antibraking system in neural development. Mol Cell 55: 482-494, 2014.

21. Cheng C, Niu C, Yang Y, Wang Y and Lu M: Expression of HAUSP in gliomas correlates with disease progression and survival of patients. Oncol Rep 29: 1730-1736, 2013.

22. Periz G, Lu J, Zhang T, Kankel MW, Jablonski AM, Kalb R McCampbell A and Wang J: Regulation of protein quality control by UBE4B and LSD1 through p53-mediated transcription. PLoS Biol 13: e1002114, 2015. 